\title{
Capture Probability and Throughput Analysis of Slotted ALOHA and Unslotted np-ISMA in a Rician/Rayleigh Environment
}

\author{
Iguh Widipangestu, Aart J. 'T Jong, and Ramjee Prasad, Senior Member, IEEE
}

\begin{abstract}
The performance of mobile radio communication systems is analyzed, in terms of the capture probability and throughput, for the slotted ALOHA and the unslotted np-ISMA (nonpersistent Inhibit Sense Multiple Access) protocol with receiver capture. The influence of Rayleigh faded interference and additive white Gaussian noise (AWGN) is studied on the performance of the two protocols considering BPSK (Binary Phase Shift Keying) modulated desired test signal, which suffers from Rician fading and the near-far effect. BCH (Bose Chaudhuri Hocquenghem) error correction codes consisting of packets with equal number of bits are introduced to improve the performance of the system, considering both fast and slow multipath fading.
\end{abstract}

\section{INTRODUCTION}

S EVERAL random multiple access protocols have been introduced in mobile radio communication systems to improve the efficiency of these systems. The performance of these systems using random multiple access protocols, such as slotted ALOHA and unslotted nonpersistent ISMA (Inhibit Sense Multiple Access), has been investigated extensively in the literature, e.g., in [1]-[12]. In [12], the BPSK (Binary Phase Shift Keying) modulated signals transmitted by both (desired) test and interfering terminals (terminals transmitting a packet at the same time) are assumed to suffer from Rayleigh fading (i.e., the mobile terminal is not within line-of-sight of the base station) due to relatively large propagation distances between base station and mobile terminals (macrocellular systems). However, because of the growing number of users in mobile communication systems, (micro) cells having smaller radii have been introduced to handle the increased amount of traffic. In several studies investigating the propagation characteristics of microcells, it has been concluded [13] that if the mobile terminal is in line-of-sight of the base station, its signal amplitude has a Rician distribution compared to a Rayleigh distribution, when there is no line-of-sight. In this paper, the test terminal is assumed to be relatively close to the base station, so a line-of-sight component is present, thus,

Manuscript received July 13, 1992; revised October 5, 1992.

I. Widipangestu was with the Telecommunication and Traffic-Control Systems Group, Delft University of Technology, 2600 GA Delft, The Netherlands. He is now with the Technology Development Division, Agency for Assessment and Application of Technology (BPPT), Jakarta, Indonesia.

A. J. 'T Jong was with the Telecommunication and Traffic-Control Systems Group, Delft University of Technology, 2600 GA Delft, The Netherlands. He is now with the Dutch Army.

R. Prasad is with the Telecommunication and Traffic-Control Systems Group, Delft University of Technology, 2600 GA Delft, The Netherlands.

IEEE Log Number 9215201. also in this case, the test terminal suffers from Rician fading. The interfering terminals are assumed to be either: 1) near to the border of the cell and therefore have larger propagation distances without line-of-sight component and suffer from Rayleigh fading, or 2) close to the base station with Rayleigh fading which is due to the nonexistence of line-of-sight between the base station and interfering terminal antennas. Thus, the signal envelope distribution of the interfering terminals is always Rayleigh fading irrespective of their positions with respect to the base station. The above assumptions are made for the simplicity of analysis to obtain initial results for the random multiple access protocols in a microcellular system.

In this paper, the performance of mobile ratio communication systems using the slotted ALOHA and the unslotted nonpersistent ISMA protocol is investigated in terms of the capture probability and the throughput. The capture probability is defined as the probability that a packet, consisting of a number of bits, is successfully transmitted, while the throughput is defined as the number of packets that are received correctly within a certain time. The effect of using $\mathrm{BCH}$ (Bose Chaudhuri Hocquenghem) error correction codes on the performance of mobile communication systems with random access techniques, investigated earlier, e.g., in [2] and [14], is also investigated. This paper is organized as follows. In Section II, the channel model, concerning all propagation and traffic characteristics of the channel, is discussed, while in Section III, the capture probability is investigated for both fast and slow multipath fading. The performance of mobile radio communication systems in terms of the throughput using two random access protocols, viz., slotted ALOHA and unslotted nonpersistent ISMA, is studied in Section IV. BCH error correction codes are introduced to the model in Section V. The user data throughput is defined and evaluated in Section VI, and, finally, conclusions are given in Section VII.

\section{Channel Model}

In the model presented in this paper, the test terminal is assumed to be very near to the base station, so the test terminal is in line-of-sight of the base station. In this way, the amplitude of the test signal can be modeled by a Rician pdf (probability density function) [15]

$$
f_{\rho_{0}}\left(\rho_{0} \mid \bar{p}_{0}\right)=\frac{\rho_{0}}{\sigma^{2}} \exp \left(-\frac{\rho_{0}^{2} / 2+s^{2} / 2}{\sigma^{2}}\right) I_{0}\left(\frac{s \rho_{0}}{\sigma^{2}}\right)
$$


where $\rho_{0}$ is the amplitude of the received instantaneous test signal, $\sigma^{2}$ is the scattered power, $s$ is the peak value of the specular component, $\bar{p}_{0}$ is the local-mean power of the test signal, with $\bar{p}_{0}=s^{2} / 2+\sigma^{2}$, and $I_{0}()$ is the modified Bessel function of the first kind and zeroth order [16]. An important parameter is the Rician parameter $K$, where $K$ is defined as the ratio of the power of the specular (line-of-sight) component and the power of the scattered (reflected) component, thus $K=s^{2} / 2 \sigma^{2}$. In this way, it is possible to express (1) in terms of the instantaneous power $p_{0}$ of the test signal (with $\left.p_{0}=\frac{1}{2} \rho_{0}^{2}\right)$ and the Rician parameter $K$

$$
\begin{aligned}
f_{p_{0}}\left(p_{0} \mid \bar{p}_{0}\right)= & \frac{(1+K)}{\bar{p}_{0}} \exp \left(-K-\frac{p_{0}(1+K)}{\bar{p}_{0}}\right) I_{0} \\
& \cdot\left(\sqrt{\frac{4 p_{0} K(1+K)}{\bar{p}_{0}}}\right) .
\end{aligned}
$$

Shadowing, consisting of very slow fluctuations of the localmean power, is not considered in this paper. The interfering terminals are assumed to be situated near the border of the cell, so a line-of-sight between any of the mobile terminals and base station is absent (i.e., $K=0$ ). In that case, the amplitude $\rho_{j}$ of the $j$ th signal $(j=1, \cdots, i$, where $i$ is the number of interfering signals) is Rayleigh distributed, while the instantaneous power $p_{j}$ is then negative exponentionally distributed about the local-mean power $\bar{p}_{j}$

$$
f_{p_{j}}\left(p_{j} \mid \bar{p}_{j}\right)=\frac{1}{\bar{p}_{j}} \exp \left(-\frac{p_{j}}{\bar{p}_{j}}\right)
$$

The local-mean powers of both test signal and interfering signals are determined by path loss effects during propagation, which means that the power of the transmitted signal decreases with increasing distance to a certain propagation loss exponent $\beta$. This propagation loss exponent $\beta$ depends on the environment where the mobile communication system is situated. In this paper, $\beta$ is assumed to be fixed, viz., $\beta=4$. In this way, the local-mean power $\bar{p}_{j}$ of the $j$ th signal (both test signal and interfering signals) at a normalized distance $r_{j}$ (normalized to the cell radius, so $0<r_{j} \leq 1$ ), with $r_{j}$ defined as the distance from terminal $j$ to the base station, is given by

$$
\bar{p}_{j}=\frac{1}{r_{j}^{4}} .
$$

A radio channel with a total traffic load $G$, expressed in packets per unit time, or packet per time slot, is considered. The terminals are assumed to move randomly in the area around the base station. If the position of the mobile terminal is unknown, the local-mean power $\bar{p}_{j}$ has the following pdf

$$
f_{\bar{p}_{j}}\left(\bar{p}_{j}\right)=\frac{2 \pi}{G} G\left(r_{j}\right) r_{j}\left|\frac{d r_{j}}{d \bar{p}_{j}}\right| .
$$

The average number of packet transmissions per unit time and per unit area at normalized distance $r_{j}$ from the base station is denoted by $G\left(r_{j}\right)$. The offered traffic $G(r)$ is given by a quasi-uniform spatial distribution function [5]

$$
G(r)=\frac{G}{\pi} \exp \left(-\frac{\pi}{4} r^{4}\right)
$$

which is an approximation of the exactly uniform distribution [12], resulting in the fact that almost all traffic arrives from normalized distances $0<r<1$. The distribution function of the received local-mean power $\bar{p}_{j}$ can then, after substituting (4) and (6) in (5), be given by

$$
f_{\bar{p}_{j}}\left(\bar{p}_{j}\right)=\frac{\bar{p}_{j}^{-(3 / 2)}}{2} \exp \left(-\frac{\pi}{4 \bar{p}_{j}}\right) .
$$

The pdf of the local-mean total interference power $\bar{p}_{t}$, caused by $i$ interfering signals, with $\bar{p}_{t}=\Sigma \bar{p}_{j}$, is determined after $i$-fold convolution of the pdf of the local-mean power, given by (7) [5]

$$
f_{\bar{p}_{t}}\left(\bar{p}_{t}\right)=\left[f_{\bar{p}_{j}}\left(\bar{p}_{j}\right)\right]^{\otimes i}=\frac{i}{2} \bar{p}_{t}^{-(3 / 2)} \exp \left(-\frac{\pi i^{2}}{4 \bar{p}_{t}}\right) .
$$

\section{CAPTURE ProbaBILITY}

In this paper, only BPSK modulation is considered. The receiver input signal $y(t)$ is of the form [4], [12]

$$
\begin{aligned}
y(t)= & \rho_{0} k_{0} \cos \left(\omega_{c} t+\phi_{0}\right) \\
& +\sum_{j=1}^{n} \rho_{j} k_{j} \cos \left(\omega_{c} t+\phi_{j}\right)+n(t)
\end{aligned}
$$

where $k_{j}$ represents phase reversals due to BPSK modulation of the $j$ th signal, and $n(t)$ represents the additive white Gaussian noise (AWGN). The received energy per bit is $E_{b}=p_{0} T_{b}=\frac{1}{2} \rho_{0}^{2} T_{b}$. For each signal, the amplitude $\rho_{j}$ and phase $\phi_{j}$ are assumed to remain constant during one bit duration $T_{b}$. Further, perfect bit synchronization is assumed, i.e., for each of the interfering signals, exactly overlapping bit periods are assumed. The receiver is also assumed to be locked to the test signal. In the receiver, $y(t)$ is multiplied by a locally generated cosine $\left(2 \cos \omega_{c} t\right)$ and integrated over the entire bit duration $T_{b}$. In a Rayleigh-fading channel, the in-phase carrier components of the $i$ interfering signals are all independent Gaussian variables, with variance $\bar{p}_{j}$ [12]. The bit error rate (BER) for a receiver locked to the BPSK modulated test signal in the presence of multiple interferers with local-mean power $\bar{p}_{t}$ and AWGN is of the form [4], [12]

$$
P_{b e}\left(b e \mid p_{0}, \bar{p}_{t}\right)=\frac{1}{2} \operatorname{erfc}\left(\sqrt{\frac{p_{0} T_{b}}{N_{0}+\bar{p}_{t} T_{b}}}\right)
$$

where erfc( ) represents the complementary error function [16] and $N_{0}$ is the (one-sided) spectral power density of the AWGN.

The radio signals are transmitted in packets, each having a number of $L$ bits. The capture probability is defined as the probability that a packet of $L$ bits is received correctly, so all $L$ bits are received without error. A capture model is discussed in [5]-[7], where a test packet captures the receiver if and only if the ratio of the power of the test packet and the total power of $i$ interfering packets exceeds a certain value called the capture ratio. Here, this capture ratio is assumed to be chosen in such a way that the receiver locks to the test packet despite the interfering packets. The capture probability is investigated in this section, where two different types of multipath fading [2] are distinguished: 1) fast multipath fading - the signal 
amplitude is assumed independent from bit to bit, the bit errors in successive bits are assumed independent and have equal BER; and 2) slow multipath fading-the amplitude and phase of each signal are assumed constant for the entire duration of the packet $L T_{b}$.

In the following analysis, it is assumed that the location of the test terminal as well as the interfering terminals follow the same spatial distribution given by (6). The performance analysis is simplified by assuming that the test and interfering signals are always Rician and Rayleigh faded, respectively, irrespective of their locations with respect to the base station. Rayleigh distribution is a particular case for Rician distribution with no line-of-sight, i.e., Rician parameter $K=0$.

\section{A. Fast Multipath Fading}

Because the instantaneous power $p_{0}$ of the test signal has to be averaged over the pdf (2) to take the (Rician) fading statistics into account, and each of the $L$ bits of the packet has equal BER, the capture probability for fast multipath fading is given by

$$
\begin{aligned}
P_{c a p}\left(\text { fast } \mid \bar{p}_{0}, \bar{p}_{t}\right)= & {\left[1-\int_{0}^{\infty} P_{b e}\left(b e \mid p_{0}, \bar{p}_{t}\right)\right.} \\
& \left.\cdot f_{p_{0}}\left(p_{0} \mid \vec{p}_{0}\right) d p_{0}\right]^{L} .
\end{aligned}
$$

If the quasi-uniform spatial distribution of the position of both test terminal and $i$ interfering terminals, given by (7) and (8), respectively, is taken into account, the following expression for the capture probability for the fast multipath fading channel is obtained

$$
\begin{gathered}
P_{\text {cap }}\left(\text { fast } \mid \bar{p}_{0}, \bar{p}_{t}\right)=\int_{0}^{\infty} \int_{0}^{\infty}\left[1-\int_{0}^{\infty} P_{b e}\left(b e \mid p_{0}, \bar{p}_{t}\right)\right. \\
\left.\cdot f_{p_{0}}\left(p_{0} \mid \bar{p}_{0}\right) d p_{0}\right]^{L} f_{\bar{p}_{0}}\left(\bar{p}_{0}\right) \\
\cdot f_{\bar{p}_{t}}\left(\bar{p}_{t}\right) d \bar{p}_{0} d \bar{p}_{t} .
\end{gathered}
$$

Introducing the variables $x, y$, and $z$, with

$$
\begin{aligned}
x & =\frac{p_{0}(1+K)}{\bar{p}_{0}}, \quad y=\sqrt{\frac{\pi i^{2}}{4 \bar{p}_{t}}}, \quad \text { and } \\
z & =\sqrt{\frac{\pi}{4 \bar{p}_{0}}} \\
d x & =\frac{(1+K)}{\bar{p}_{0}} d p_{0}, \quad d y=-\frac{2 y^{3}}{\pi i^{2}} d \bar{p}_{t}, \quad \text { and } \\
d z & =-\frac{2 z^{3}}{\pi} d \bar{p}_{0}
\end{aligned}
$$

finally, the capture probability for the fast multipath fading channel becomes, after some manipulations,

$$
\begin{aligned}
& P_{c a p}\left(\text { fast } \mid \bar{p}_{0}, \bar{p}_{t}\right) \\
& =\int_{0}^{\infty} \int_{0}^{\infty}\left[1-\frac{\exp (-K)}{2} \int_{0}^{\infty} \exp (-x) I_{0}(\sqrt{4 K x}) \operatorname{erfc}\right.
\end{aligned}
$$

$$
\begin{aligned}
& \left.\left(\sqrt{\frac{z^{2}(1+K)\left(\frac{4 N_{0}}{T_{b}}+\frac{\pi i^{2}}{y^{2}}\right)}{n}}\right) d x\right]^{L} \\
& \cdot \frac{4}{\pi} \exp \left(-y^{2}-z^{2}\right) d y d z .
\end{aligned}
$$

\section{B. Slow Multipath Fading}

After averaging $p_{0}$ over the pdf (2), the capture probability for the slow multipath fading channel is given by

$$
\begin{aligned}
P_{\text {cap }}\left(\text { slow } \mid \bar{p}_{0}, \bar{p}_{t}\right) & \\
= & \int_{0}^{\infty}\left[1-P_{b e}\left(b e \mid p_{0}, \bar{p}_{t}\right)\right]^{L} \\
& \cdot f_{p_{0}}\left(p_{0} \mid \bar{p}_{0}\right) d p_{0} .
\end{aligned}
$$

In the same way as for the fast multipath fading channel, the capture probability for the slow multipath fading channel can be found by taking the quasi-uniform spatial distribution of the position of both test terminal and $i$ interfering terminals, (7) and (8), respectively, into account

$$
\begin{aligned}
P_{c a p}\left(\operatorname{slow} \mid \bar{p}_{0}, \bar{p}_{t}\right) & \exp (-K) \int_{0}^{\infty} \int_{0}^{\infty} \int_{0}^{\infty}\left[1-\frac{1}{2} \operatorname{erfc}\right. \\
& \left.\cdot\left(\sqrt{\frac{\pi x}{z^{2}(1+K)\left(\frac{4 N_{0}}{T_{b}}+\frac{\pi i^{2}}{y^{2}}\right)}}\right)\right]^{L} \\
& \cdot \frac{4}{\pi} \exp \left(-x-y^{2}-z^{2}\right) \\
& \cdot I_{0}(\sqrt{4 K x}) d x d y d z .
\end{aligned}
$$

Computational results for the capture probability in both fast and slow multipath fading channels are given in Figs. 1-3. In Fig. 1, the capture probability for the fast multipath fading channel as a function of the normalized distance is given for several values of the number of interferers. The capture probability for fast multipath fading is given in (14); however, in Fig. 1, it is assumed that only the positions of the $i$ interfering terminals have a quasi-uniform spatial distribution (8), while the test terminal is at distance $r$ from the base station. This means that the capture probability $(11)$ only has to be averaged over the quasi-uniform spatial pdf of $\bar{p}_{t}(8)$. In Fig. $1, K=7 \mathrm{~dB}$, the signal-to-noise ratio (SNR) $\bar{p}_{o} /\left(N_{o} / T_{b}\right)$ is equal to $10 \mathrm{~dB}$ and $L=63$ bits. From Fig. 1 , it can be seen that the number of interfering terminals has a large influence on the capture probability: the capture probability decreases as the number of interferers increases.

Fig. 2 shows the influence of the SNR and packet length on the capture probability for fast multipath fading (14) as a function of the number of interferers $i$. Also, in Fig. $2, K=7$ $\mathrm{dB}$ is assumed. An increasing number of interfering terminals causes the capture probability to decrease dramatically. From 


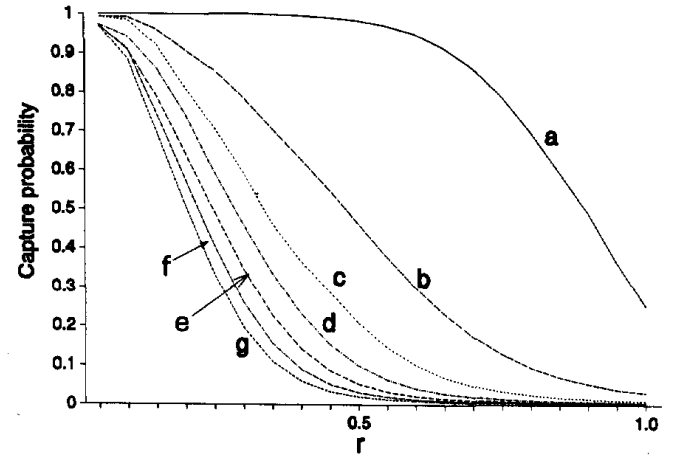

Fig. 1. Capture probability as a function of normalized distance for fast multipath fading, $K=7 \mathrm{~dB}$, SNR $=10 \mathrm{~dB}$, and $L=63$ bits for $i=0,1, \cdots, 6$ interferers (a-g, respectively).

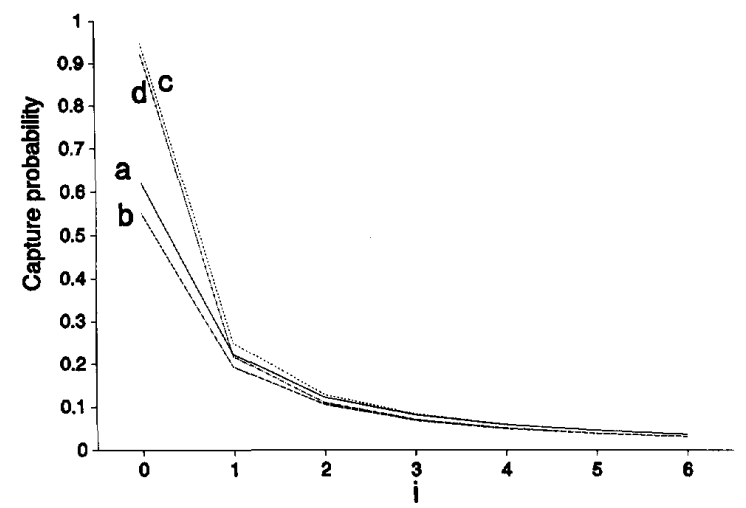

Fig. 2. Capture probability as a function of the number of interferers for fast multipath fading and $K=7 \mathrm{~dB}$, for $L=63$ bits (a: SNR $=10 \mathrm{~dB}$, c: SNR $=40 \mathrm{~dB}$ ), and $L=127$ bits (b: SNR $=10 \mathrm{~dB}$, d: SNR $=40 \mathrm{~dB}$ ).

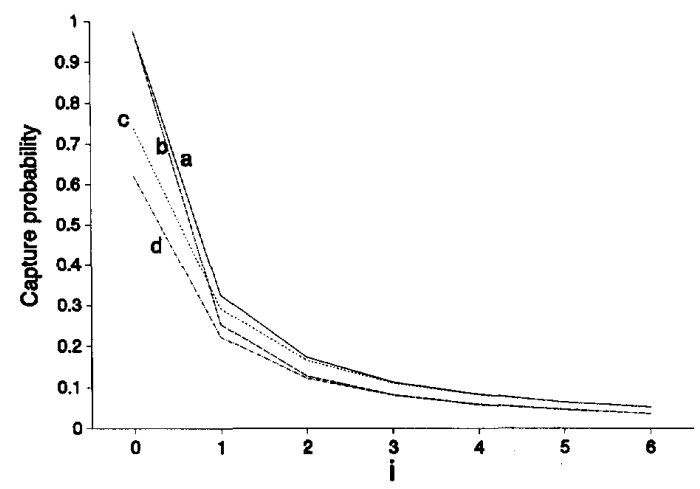

Fig. 3. Capture probability as a function of the number of interferers, with $K=7 \mathrm{~dB}$ and $L=63$ bits, for slow (a: $S N R=40 \mathrm{~dB}$, c: $S N R=10 \mathrm{~dB}$ ), and fast multipath fading (b: SNR $=40 \mathrm{~dB}, \mathrm{~d}:$ SNR $=10 \mathrm{~dB}$ ).

Fig. 2, it can also be seen that the SNR has a larger influence on the capture probability than $L$, especially for large SNR and small $i$. It is worth mentioning here that the SNR represents the local-mean signal-to-noise ratio $\bar{p}_{o} /\left(N_{o} / T_{b}\right)$. Therefore, large SNR yields enhanced throughput because of the reduced BER. For $i>6$, the number of interfering signals has a negligible influence on the capture probability because the capture probability remains almost constant.

A comparison between the capture probabilities for fast and slow multipath fading channels, given by (14) and (16), respectively, is given in Fig. 3. In Fig. 3, the capture probability is plotted as a function of the number of interfering terminals for different SNR. The difference between fast and slow multipath fading (slow multipath fading gives better performance) is relatively large for small $i$ and small SNR, as can be seen in Fig. 3. For $i>3$, the performance for fast and slow multipath fading is almost equal, and only SNR has a small influence on the capture probability.

\section{RANDOM ACCESS SySTEMS With ReCEIVER CAPTURE}

Random access techniques are introduced in mobile communications to facilitate the users. In random access systems, users transmit whenever they want to, with possibly a few constraints depending on the particular access method used. Several random access protocols are known, but in this paper the performance of mobile radio communication systems using the slotted ALOHA and the unslotted nonpersistent ISMA protocol is studied and compared.

\section{A. Slotted ALOHA}

Using the slotted ALOHA protocol, terminals transmit their packets regardless of other terminals which might already be transmitting a packet in the same time slot, where a time slot has the same length as the length of the packet. If two (or more) packets are being transmitted at the same time, the packets get lost. An unsuccessful transmission of a packet is discovered by the terminal, if, after a certain time, no acknowledgment from the transmitter has been received. After a random time, the terminal retransmits the same packet. On channels with receiver capture, the packet with the strongest signal has still the probability of being "captured" by the receiver, also when more packets are being transmitted at the same time. The probability $P_{i}(i)$ that the test packet suffers from $i$ other packets in the same time slot is assumed to be Poisson distributed, with mean $G$,

$$
P_{i}(i)=\frac{G^{i}}{i !} \exp (-G) \text {. }
$$

The throughput $S$ of slotted ALOHA with receiver capture can then be found by multiplying the capture probability by $P_{i}(i)$

$$
\begin{aligned}
& S(\text { slotted ALOHA }) \\
& =G \sum_{i=0}^{\infty} P_{i}(i) P_{\text {cap }}\left(\text { fast } / \text { slow } \mid \bar{p}_{0}, \bar{p}_{t}\right) \\
& =G \sum_{i=0}^{\infty} \frac{G^{i}}{i !} \exp (-G) \\
& \cdot P_{\text {cap }}\left(\text { fast } / \text { slow } \mid \bar{p}_{0}, \bar{p}_{t}\right) .
\end{aligned}
$$

The throughput for the fast and the slow multipath fading channel can be found by substituting the appropriate capture probability in (18), given by (14) and (16), respectively. 


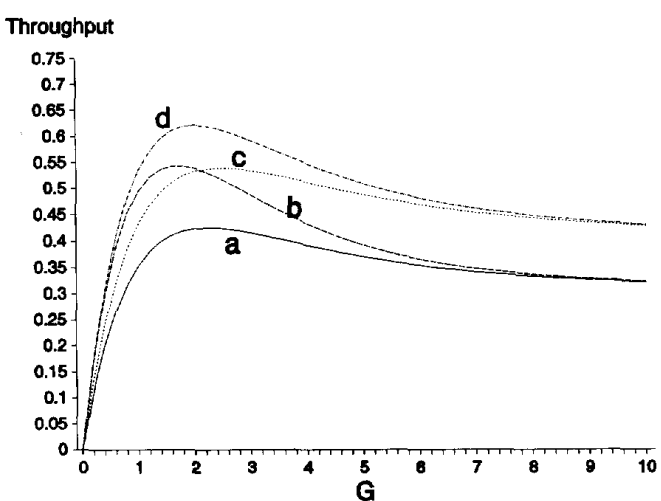

Fig. 4. Throughput of slotted ALOHA as a function of $G$, for $L=63$ bits and $K=7 \mathrm{~dB}$, for fast multipath fading (a: $S N R=10 \mathrm{~dB}, \mathrm{~b}: \mathrm{SNR}=40 \mathrm{~dB}$ ), and slow multipath fading $(\mathrm{c}: \mathrm{SNR}=10 \mathrm{~dB}, \mathrm{~d}: \mathrm{SNR}=40 \mathrm{~dB})$.

Computational results for the throughput of slotted ALOHA are given in Figs. 4 and 5. In Fig. 4, the throughput is given as a function of the total traffic load $G$, for fast and slow multipath fading and different values of SNR. In Fig. 4, $K=7 \mathrm{~dB}$ and $L=63$ bits are assumed. From Fig. 4 , it can be seen that slow multipath fading gives a much higher performance than fast multipath fading. The SNR only influences the throughput for $G<10$.

Fig. 5 shows the effect of $K$ on the throughput. Propagation measurements in several environments, for instance, in [13], resulted in several values of $K(K=0$ corresponds to Rayleigh fading, i.e., the line-of-sight component is absent). In Fig. 5, the throughput for fast multipath fading is given as a function of $G$, with $L=63$ bits and SNR $=10 \mathrm{~dB}$. The Rician parameter $K$ has a very large influence on the throughput. For large $K$, the performance is much better than for $K=0$; thus, if the test terminal is in line-of-sight of the base station, the performance is much better than when there is no line-ofsight. This means that if the test terminal is very near to the base station (a strong line-of-sight component), the probability that a packet is successfully transmitted (or captured by the receiver) is higher than for the case the distance between test terminal and base station is larger (a smaller line-of-sight component).

\section{B. Unslotted np-ISMA}

According to the nonpersistent ISMA protocol, each terminal must listen to the channel before transmitting. Because the protocol is nonpersistent, the terminals do not continuously sense, but wait a random period of time. An advantage of the ISMA protocol is that ISMA eliminates the hidden-terminal problem [6], [10], [11] occurring in the CSMA (Carrier Sense Multiple Access) protocol, where terminals might not be able to sense a transmission by another terminal. The base station transmits a "busy" signal on the outbound channel as soon as an inbound packet is being received. After complete reception of the packet, the "busy" signal is removed, so that the channel becomes "idle." In ISMA, collisions may occur due to new packet transmissions during the transmission delay of the inhibit signal $d$, necessary to switch from "busy" to "idle"

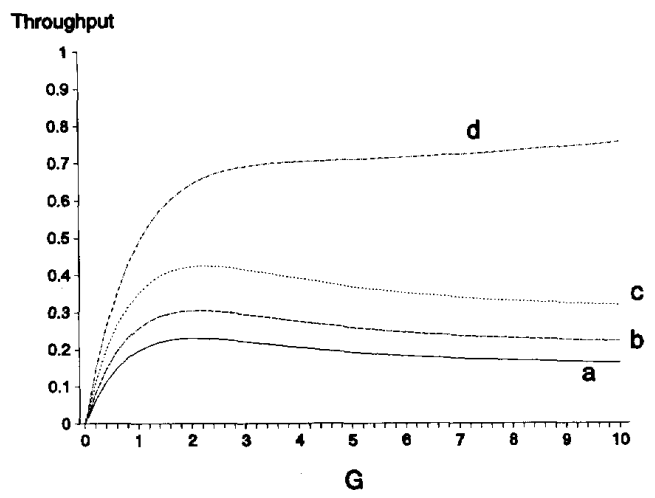

Fig. 5. Throughput of slotted ALOHA as a function of $G$, for fast multipath fading, $L=63$ bits, and SNR $=10 \mathrm{~dB}$, for (a) $K=0$ (Rayleight fading), (b) $K=3 \mathrm{~dB}$, (c) $K=7 \mathrm{~dB}$, and (d) $K=10 \mathrm{~dB}$.

(and reverse). This delay is normalized to the packet length, resulting in $0 \leq d<1$.

The probability $U$ that the channel, during a cycle (one cycle consists of an idle period plus a transmission period), is used without conflicts is defined as the probability that a test packet is overlapped by $i$ interfering packets $P_{i}(i)$, multiplied by the probability that the test packet is received correctly in the presence of $i$ interfering packets

$$
\begin{aligned}
U= & \sum_{i=0}^{\infty} P_{i}(i)(i+1) P_{c a p}\left(\text { fast } / \text { slow } \mid \bar{p}_{0}, \bar{p}_{t}\right) \\
& \text { with } \quad P_{i}(i)=\frac{(d G)^{i}}{i !} \exp (-d G) .
\end{aligned}
$$

The term $(i+1)$ is included in (19), because when inhibit bits are in the outbound channel and a test packet is subjected to $i$ interfering packets, $i+1$ packets are presented during the busy period. One of these $i+1$ packets is capable of capturing the receiver with a certain probability. One must take care of the fading type of the test and interfering packets while evaluating the success probability $U$ using (19). In case of Rayleigh faded test and interfering packets, $P_{\text {cap }}$ (fast/slow $/ \bar{p}_{0}, \bar{p}_{t}$ ) can be calculated using (14) and (16) by substituting $K=0$. If the test packet is Rician faded and the interfering packets are Rayligh faded, then a proper value of $K$ is used in (14) and (16) to calculate the success probability. Thus, it is clear that the expression for the success probability changes with the change in fading type.

The throughput for unslotted np-ISMA with capture can then be found by dividing $U$ by the sum of the expected duration of the transmission period $T$, given by [10], [11]

$$
T=1+2 d-\frac{1}{G}[1-\exp (-d G)]
$$

and the expected duration of the idle period $I$, with $I=1 / G$; thus,

$$
\begin{gathered}
S \text { (unslotted np-ISMA) } \\
=\frac{U}{T+I}
\end{gathered}
$$




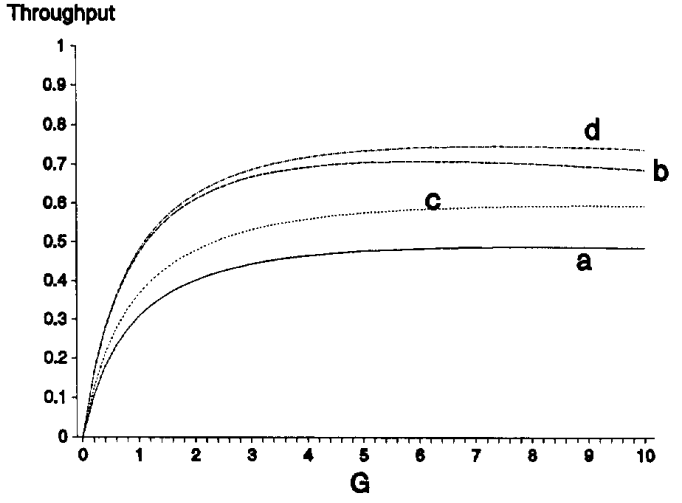

Fig. 6. Throughput of unslotted np-ISMA as a function of $G$, for $L=63$ bits, $K=7 \mathrm{~dB}$, and $d=0.05$ for fast multipath fading (a: $S N R=10 \mathrm{~dB}$, b: SNR $=40 \mathrm{~dB}$ ), and slow multipath fading (c: $S N R=10 \mathrm{~dB}, \mathrm{~d}: \mathrm{SNR}=40 \mathrm{~dB}$ ).

$$
\begin{gathered}
=\frac{\sum_{i=0}^{\infty} \frac{(d G)^{i}}{i !} \exp (-d G)(i+1)}{(1+2 d)+\frac{1}{G} \exp (-d G)} \\
\cdot P_{\text {cap }}\left(\text { fast } / \text { slow } \mid \bar{p}_{0}, \bar{p}_{t}\right)
\end{gathered}
$$

where, again, the throughput for fast and slow multipath fading channels can be found by substituting the capture probability (14) and (16), respectively, in (21).

Computational results for the throughput of unslotted npISMA are given in Fig. 6. In Fig. 6, the throughput for both fast and slow multipath fading is given as a function of $G$ for different values of SNR, with $K=7 \mathrm{~dB}, L=63$ bits, and $d=0.05$. From Fig. 6 , it can be seen that, also for ISMA, slow multipath fading gives a higher throughput than fast multipath fading. A higher value of SNR also gives a higher throughput for all values of $G$.

Fig. 7 compares the throughput for the slotted ALOHA protocol and the unslotted np-ISMA protocol. The throughput for fast multipath fading is given as a function of $G$. In Fig. 7 , again $L=63$ bits and $K=7 \mathrm{~dB}$ are assumed. From Fig. 7 , it can be seen that, at least for high values of $G$, the unslotted np-ISMA protocol gives a much higher throughput than the slotted ALOHA protocol. It can also be seen that a larger value of $d$ gives a lower performance.

\section{ERROR CORRECTION CODING}

In Section III, it was discussed that all $L$ bits of the packet have to be received correctly in order to have a successful reception of the packet. At this point, error correction coding is added to the system to improve the performance. Using error correction coding some of the $L$ bits may be received incorrectly, still resulting in a successful reception of the packet. In this paper, only linear $\mathrm{BCH}$ error correction codes with parameters $(L, k, t)[17]$ are used, where $L$ is the packet length, $k$ is the number of actual information ("user") bits in the packet (or codeword), and $t$ is the error correction capability. This error correction capability offers the possibility of correcting up to $t$ erroneous bits. This means that to receive the packet successfully, only up to $t$ bits may be

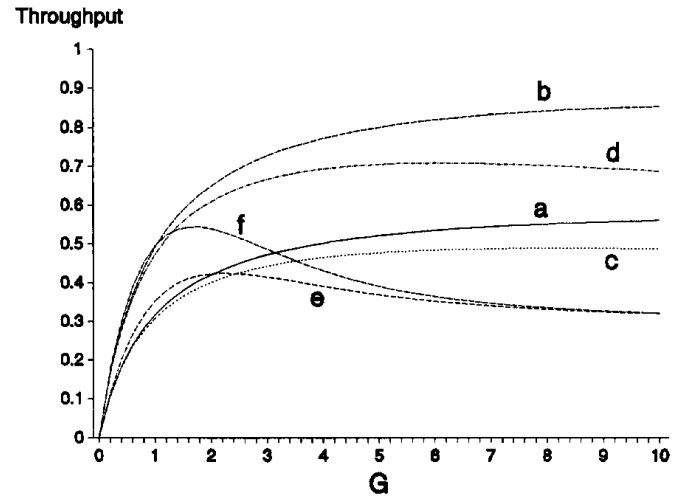

Fig. 7. Throughput as a function of $G$, for fast multipath fading, $L=63$ bits, and $K=7 \mathrm{~dB}$ for unslotted np-ISMA with $d=0.01$ (a: SNR $=10 \mathrm{~dB}$, b: SNR $=40 \mathrm{~dB}$ ), and $d=0.05(\mathrm{c}: \mathrm{SNR}=10 \mathrm{~dB}$, d: SNR $=40 \mathrm{~dB}$ ), and slotted ALOHA (e: SNR $=10 \mathrm{~dB}, \mathrm{f}: \mathrm{SNR}=40 \mathrm{~dB}$ ).

received incorrectly (instead of $t=0$, when no error correction coding is used). For a higher error correction capability, the number of parity-check bits increases, resulting in a lower code efficiency because $k$ diminishes. Thus, a tradeoff between high error correction capability and high code efficiency has to be made. Usually, error correction codes also offer a certain error detection capability, denoting the number of erroneous bits the receiver can detect but not correct. The effect of the error detection capability on the performance, studied, for example, in [14], is not considered here. The probability of successful reception of a packet is given by [14]

$$
\begin{gathered}
P\left(\text { success } \mid p_{0}, \bar{p}_{t}\right) \\
=\sum_{m=0}^{t}\left(\begin{array}{c}
L \\
m
\end{array}\right)\left(P_{b e}\left(b e \mid p_{0}, \bar{p}_{t}\right)\right)^{m} \\
\cdot\left(1-P_{b e}\left(b e \mid p_{0}, \bar{p}_{t}\right)\right)^{L-m} .
\end{gathered}
$$

The expressions for the throughput of the two protocols now change because of the introduction of error correction codes.

\section{A. Slotted ALOHA}

The probability of successful reception of a packet (22) is equal to the capture probability if the appropriate BER is substituted into (22). In this way, the following expression for the throughput of slotted ALOHA is given by

$$
\begin{aligned}
& S \text { (slotted ALOHA) } \\
& =G \sum_{i=0}^{\infty} \sum_{m=0}^{t}\left(\begin{array}{c}
L \\
m
\end{array}\right) \frac{G^{i}}{i !} \\
& \cdot \exp (-G) P_{\text {cap }}\left(\text { fast } / \text { slow } \mid \bar{p}_{0}, \bar{p}_{t}\right)
\end{aligned}
$$

where the capture probability for fast and slow multipath fading with coding is given by

$$
\begin{aligned}
P_{\text {cap }}\left(\text { fast } \mid \bar{p}_{0}, \bar{p}_{t}\right) & \\
= & \int_{0}^{\infty} \int_{0}^{\infty} \\
& \cdot\left(1-\int_{0}^{\infty} P_{b e}\left(b e \mid p_{0}, \bar{p}_{t}\right) f_{x}(x) d x\right)^{L-m}
\end{aligned}
$$




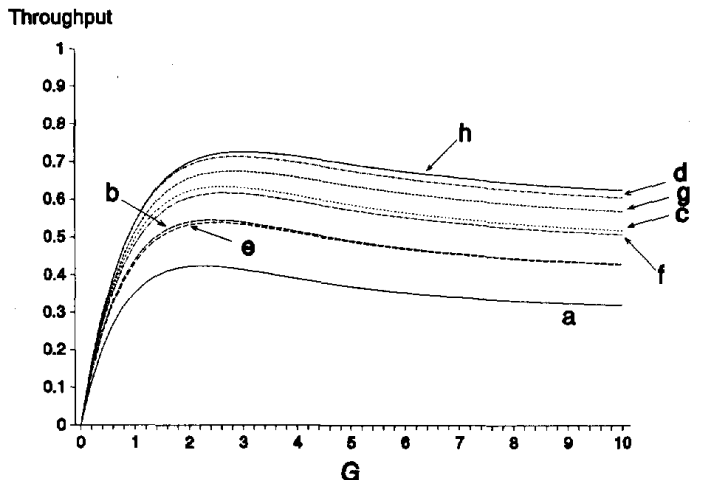

Fig. 8. Throughput of slotted ALOHA with coding as a function of $G$, for SNR $=10 \mathrm{~dB}, K=7 \mathrm{~dB}$, and $L=63$ bits, for fast multipath fading with $t=0,1,2$, and 3 (a-d, respectively) and slow multipath fading with $t=0,1,2$, and 3 (e-h, respectively)

$$
\begin{aligned}
& \cdot\left(\int_{0}^{\infty} P_{b e}\left(b e \mid p_{0}, \bar{p}_{t}\right) f_{x}(x) d x\right)^{m} \\
& \cdot f_{y}(y) f_{z}(z) d y d z
\end{aligned}
$$

and

$$
\begin{aligned}
P_{\text {cap }}\left(\operatorname{slow} \mid \bar{p}_{0}, \bar{p}_{t}\right) & \\
= & \int_{0}^{\infty} \int_{0}^{\infty} \int_{0}^{\infty}\left(1-P_{b e}\left(b e \mid p_{0}, \bar{p}_{t}\right)^{L-m}\right. \\
& \cdot\left(P_{b e}\left(b e \mid p_{0}, \bar{p}_{t}\right)\right)^{m} f_{x}(x) f_{y}(y) \\
& \cdot f_{z}(z) d x d y d z,
\end{aligned}
$$

respectively.

Computational results for the throughput of slotted ALOHA with error correction coding are shown in Fig. 8. Fig. 8 shows the effect of the error correction capability $t$ on the throughput of the fast multipath fading channel. The throughput is plotted as a function of $G$, with $K=7 \mathrm{~dB}, L=63$ bits, and SNR $=10 \mathrm{~dB}$. From Fig. 8, it can be seen that a larger value of $t$ gives also a higher throughput. For larger values of $t$, the difference between the performance of fast and slow multipath fading decreases; however, slow multipath fading still gives a better performance than fast multipath fading.

\section{B. Unslotted np-ISMA}

As for the slotted ALOHA protocol, the expression for the throughput of unslotted np-ISMA changes due to the introduction of error correction codes

$$
\begin{array}{r}
S(\text { unslotted np-ISMA) } \\
=\frac{\sum_{i=0}^{\infty} \sum_{m=0}^{t}\left(\begin{array}{c}
L \\
m
\end{array}\right) \frac{d G^{i}}{i !} \exp (-d G)(i+1)}{(1+2 d)+\frac{1}{G} \exp (-d G)} \\
\cdot P_{\text {cap }}\left(\text { fast } / \text { slow } \mid \bar{p}_{0}, \bar{p}_{t}\right)
\end{array}
$$

where the capture probability for fast and slow multipath

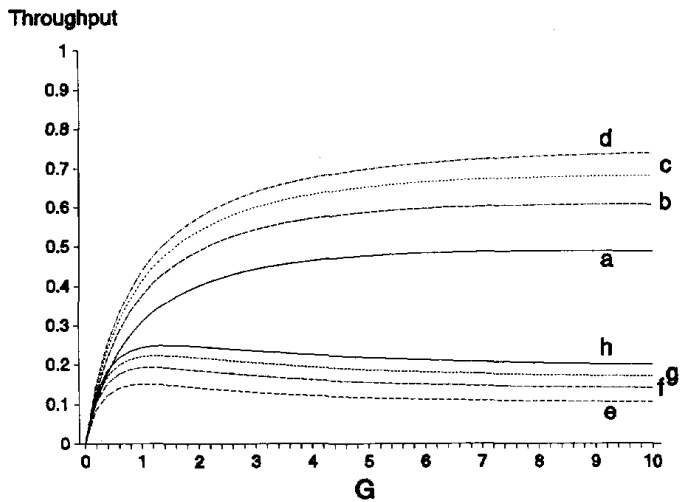

Fig. 9. Throughput of unslotted np-ISMA with coding as a function of $G$, for fast multipath fading, $\mathrm{SNR}=10 \mathrm{~dB}, L=63$ bits, and $K=7 \mathrm{~dB}$, for $d=0.05$ with $t=0,1,2$, and 3 (a-d, respectively) and $d=1$ with $t=0,1,2$, and 3 (e-h, respectively).

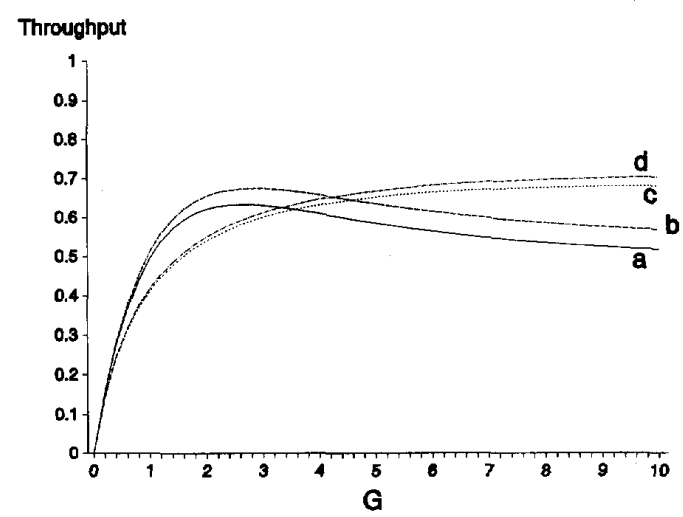

Fig. 10. Throughput as a function of $G$, for $\mathrm{SNR}=10 \mathrm{~dB}, k=51$ bits, $K=7 \mathrm{~dB}$, and $t=2$, for slotted ALOHA (a: fast, b: slow multipath fading) and unslotted np-ISMA with $d=0.05$ (c: fast, d: slow multipath fading).

fading channels with coding is given by (24) and (25), respectively.

Computational results for the throughput of unslotted $\mathrm{np}$ ISMA with error correction coding are given in Figs. 9 and 10. The throughput for fast multipath fading as a function of $G$ is plotted in Fig. 9, for several values of $d$ and $t$. In Fig. 9, again, $K=7 \mathrm{~dB}, L=63$ bits, and $\mathrm{SNR}=10 \mathrm{~dB}$ are assumed. As for the slotted ALOHA protocol, a higher value of $t$ gives a higher throughput while, again, it can be seen that a higher value of $d$ gives a small throughput.

Finally, Fig. 10 shows a comparison between the performance of slotted ALOHA and unslotted np-ISMA. In Fig. 10, the throughput for both protocols is plotted as a function of $G$, for both fast and slow multipath fading, with $K=7 \mathrm{~dB}$, $L=63$ bits, SNR $=10 \mathrm{~dB}, t=2$, and $d=0.05$. From Fig. 10, it can be seen that unslotted np-ISMA gives a higher throughput than slotted ALOHA, provided $G$ is relatively large. For the used parameters, it can also be seen that the difference in performance between fast and slow multipath fading is larger for slotted ALOHA than for unslotted npISMA. 


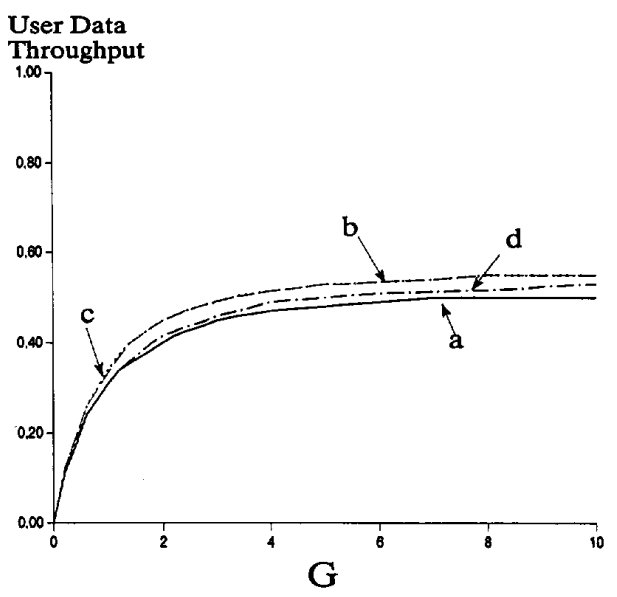

Fig. 11. User data throughput of unslotted np-ISMA with coding as a function of $G$ for fast multipath fading, SNR $=10 \mathrm{~dB}, K=7 \mathrm{~dB}$, for $d=0.05$ with $k=, 63,57,51$ and 45 (a-d respectively).

\section{USER DATA THROUGHPUT}

The user throughput $S_{u}$, i.e., the throughput of user data, is defined as the product of the throughput $S$, the user bits in a packet or a codeword $k$, and the inverse of the packet length $L$.

$$
S_{u} \triangleq S \frac{k}{L}
$$

The user data throughput $S_{u}$ of np-ISMA protocol has been calculated using the $\mathrm{BCH}(L, k, t)$ error correction codes and depicted in Figs. 11 and 12. Fig. 11 shows that with the increase in error correction capability $t$, the user data throughput $S_{u}$ first increases (curves a and b), then remains almost constant (curves $\mathrm{b}$ and $\mathrm{c}$ ), and finally decreases (curves c and d). In contrast to the user data throughput $S_{u}$, the throughput $S$ always increases with the increase in $t$ (Fig. 9). Fig. 12 compares the throughput $S$ to the user data throughput $S_{u}$. It can be seen from Fig. 12 that the throughput $S$ is higher than the user data throughput $S_{u}$. It is because $k$ is always smaller than $L$ for any value of $t$ except for $t=0, S_{u}$ and $S$ are equal. Table I shows some values for $L, k, t$ for $\mathrm{BCH}$ codes.

\section{CONCLUSIONS}

In this paper, a model is developed for the performance evaluation of mobile radio communication systems using the slotted ALOHA and the unslotted np-ISMA protocol with receiver capture. Compared to earlier studies, here the system is assumed to be in a Rician/Rayleigh environment with AWGN, where the test terminal is very near to the base station and the interfering terminals are close to the border of the cell. The amplitude of the BPSK modulated test signal is modeled by a Rician pdf, because the test terminal is assumed to be in line-of-sight of the base station, while the signals of the interfering terminals are Rayleigh distributed without line-of-sight propagation. The performance of mobile radio communication systems is measured in terms of the capture probability and the throughput. Both fast and slow multipath

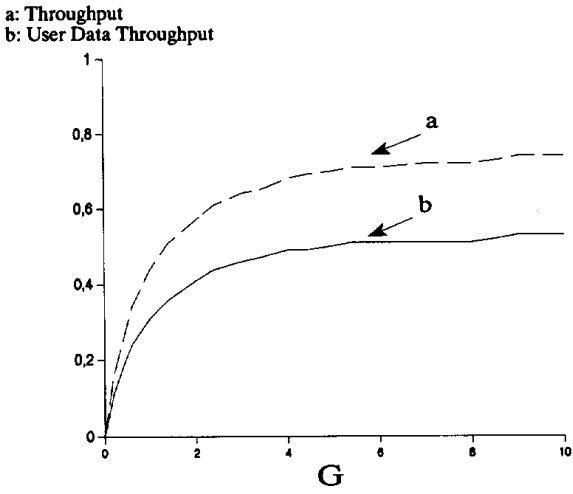

Fig. 12. (a) Throughput $S$ and (b) user data throughput $S_{u}$ as a function of $G$, for SNR $=10 \mathrm{~dB}, k=45$ bits, $K=7 \mathrm{~dB}$, and $t=3$ for fast multipath fading and np-ISMA.

TABLE I

SElected BCH $(L, k, t)$ CODES

\begin{tabular}{|c|c|c|}
\hline$L$ & $k$ & $t$ \\
\hline 63 & 63 & 0 \\
\hline 63 & 57 & 1 \\
\hline 63 & 51 & 2 \\
\hline 63 & 45 & 3 \\
\hline 31 & 21 & 2 \\
\hline 15 & 7 & 2 \\
\hline
\end{tabular}

fading channels are considered. Also, the effect of $\mathrm{BCH}$ error correction codes on the performance is investigated.

It can be concluded that the capture probability decreases dramatically if the number of interfering signal increases, however, for $i>6$, the capture probability is very small and remains almost constant. The packet length $L$ also influences the capture probability: the capture probability decreases as $L$ increases. Slow multipath fading channels offer a better performance than fast multipath fading channels. It is also concluded that for a higher value of the Rician parameter $K$ (thus when the line-of-sight component is stronger or when the test terminal is very close to the base station), the performance is very much increased. Unslotted np-ISMA gives a higher throughput than slotted ALOHA, provided the transmission delay $d$ is small and the traffic load $G$ is large. For larger values of $d$, the performance of unslotted np-ISMA decreases very rapidly. Error correction codes enhance the performance of the system because a larger error correction capability $t$ gives a higher throughput.

It is recommended to extend the present study by developing a model to include Rician fading interfering signals and the influence of multiple cells. The results obtained in this paper may be useful for both outdoor and indoor data communications.

\section{ACKNOWLEDGMENT}

The authors would like to thank the anonymous reviewers for valuable suggestions. 


\section{REFERENCES}

[1] A. U. H. Sheikh, Y.-D. Yao, and X. Wu, "The ALOHA systems in shadowed mobile radio channels with slow or fast fading," IEEE Trans. Veh. Technol., vol. 39, pp. 289-298, Nov. 1990.

[2] I. M. I. Habab, M. Kavehrad, and C.-E. W. Sundberg, "ALOHA with capture over slow and fast fading radio channels with coding and diversity," IEEE J. Select. Areas Commun., vol. 7, pp. 79-88, Jan. 1989.

[3] K. Zang and K. Pahlavan, "Relation between transmission and throughput of slotted ALOHA local packet radio networks," IEEE Trans. Commun., vol. 40, pp. 577-583, Mar. 1992.

[4] J. P. M. G. Linnartz, "Effect of fading and interference in narrowband land-mobile networks," Ph.D. dissertation, Delft Univ. Technol., The Netherlands, Dec. 1991.

[5] J. C. Ambak and W. van Blitterswijk, "Capacity of slotted ALOHA in Rayleigh fading channels," IEEE J. Select. Areas Commun., vol. SAC-5, pp. 261-269, Feb. 1987.

[6] R. Prasad and J. C. Arnbak, "Capacity analysis of non-persistent inhibit sense multiple access in channels with multipath fading and shadowing," in Proc. 1989 Workshop Mobile Cordless Telephone Commun., King's College, Univ. London, Sept. 1989, pp. 29-134.

[7] K. J. Zdunek, D. R. Ucci, and J. L. Locicero, "Throughput of nonpersistent inhibit sense multiple access with capture," Electron. Lett. vol. 25, no. 1, pp. 30-32, Jan. 5, 1989.

[8] L. Kleinrock and F. A. Tobagi, "Packet switching in radio channels: Part I-Carrier sense multiple access modes and their throughput delay characteristics," IEEE Trans. Commun., vol. COM-23, pp. 1400-1416, Dec. 1975.

[9] C. Namislo, "Analysis of mobile radio slotted ALOHA networks," IEEE J. Select. Areas Commun., vol. SAC-5, pp. 583-588, July 1984.

[10] R. Prasad, "Throughput analysis of non-persistent ISMA in multipath fading and shadowing channels," ETT, vol. 2, no. 3, pp. 313-317, May-June 1991.

[11] _ "Performance analysis of mobile packet radio networks in real channels with ISMA," IEE Proc. I, vol. 138, no. 5, pp. 458-464, Oct. 1991

[12] J. P. M. G. Linnartz, R. Hekmat, and R. J. Venema, "Near far effects in land mobile random access networks with narrow-band Rayleigh fading channels," IEEE Trans. Veh. Technol., vol. 41, Feb. 1992.

[13] R. J. C. Bultitude and G. K. Bedal, "Propagation characteristics on microcellular urban mobile radio channels at $910 \mathrm{MHz}$," IEEE J. Select. Areas Commun., vol. 7, pp. 31-39, Jan. 1989.

[14] J. P. M. G. Linnartz and J. J. P. Werry, "Error correction and error detection coding in a fast fading narrowband slotted ALOHA network with BPSK modulation," in Proc. 1st Int. Symp. Commun. Theory Appl., Crieff Hydro Hotel, Scotland, Sept. 9-13, 1991, Paper 37

[15] J. G. Proakis, Digital Communications. New York: McGraw-Hill, 1983.

[16] M. Abramowitz and I. A. Stegun, Handbook of Mathematical Functions. New York: Dover, 1965.

[17] S. Lin and D. J. Costello, Jr., Error Control Coding, Fundamentals and Applications. Englewood Cliffs, NJ: Prentice-Hall, 1983.

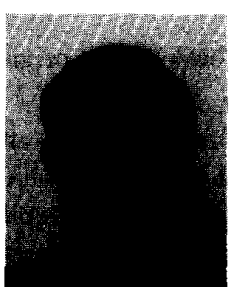

Iguh Widipangestu was born in Padang, Indonesia, in 1967. He received the Ir. (M.Sc.) degree in electrical engineering in 1992 from Delft University of Technology, The Netherlands.

Since 1992 he has been with the Technology Development Division of the Agency for Assessment and Application of Technology (BPPT) in Jakarta, Indonesia. His prime research interest includes mobile radio communication systems.

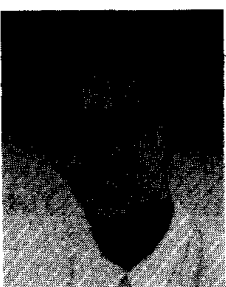

Aart J. 'T Jong was born in Sliedrecht, The Netherlands, on March 26, 1969. He attended Atheneum $\beta$ at "De Lage Waard" Papendrecht. He received the Ir. (M.Sc.) degree in electrical engineering from Delft University of Technology, The Netherlands, in June 1992.

During his study he joined the Telecommunications and Traffic-Control Group at Delft University of Technology in 1990, where he became involved in the research on mobile cellular radio communications. His thesis dealt with the performance of personal communication systems. Since July 1992 he has been serving the Dutch Army to fulfill his military service requirement in The Netherlands. His research interests are in the field of mobile radio communications.

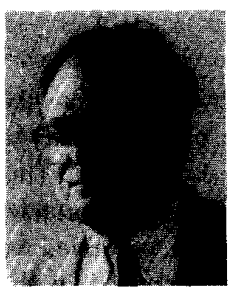

Ramjee Prasad (M'88-SM'90) was born in Babhnaur (Gaya), Bihar, India, on July 1, 1946. He received the B.Sc. (Eng.) degree from Bihar Institute of Technology, Sindri, India, and the M.Sc. (Eng.) and $\mathrm{Ph} . \mathrm{D}$. degrees from Birla Institute of Technology (BIT), Ranchi, India, in 1968, 1970, and 1979 . respectively.

He joined BIT as a Senior Research Fellow in 1970 and became an Associate Professor in 1980. During 1983-1988 he was with the University of Dar es Salaam (UDSM), Tanzania, where he became a Professor in Telecommunications at the Department of Electrical Engineering in 1986. Since February 1988 he has been with the Telecommunications and Traffic-Control Systems Group, Delft University of Technology, The Netherlands, where he is actively involved in the area of mobile, indoor, and personal radio communications. While he was with BIT, he supervised many research projects in the area of Microwave and Plasma Engineering. At UDSM he was responsible for the collaborative project "Satellite Communications for Rural Zones" with Eindhoven University of Technology, The Netherlands. He has published over 150 technical papers. His current research interest lies in packet communications, adaptive equalizers, spread-spectrum CDMA systems, and multimedia communications.

Dr. Prasad has served as a member of advisory and program committees of several IEEE international conferences. He has also presented tutorials on Mobile and Indoor Radio Communications at various universities, technica institutions and IEEE conferences. $\mathrm{He}$ is also a member of a working group of European cooperation in the field of scientific and technical re search (COST-231) project dealing with "Evolution of Land Mobile Radio (including personal) Communications" as an expert for The Netherlands He was Organizer and Interim Chairman of the IEEE Vehicular Technology/Communications Society Joint Chapter, Benelux Section. Now he is the elected Chairman of the joint chapter. He is also founder of the IEEE Symposium on communications and Vehicular Technology (SCVT) in the Benelux, and he was the Symposium Chairman of SCVT'93. He is one of the Editors-in-Chief of a new journal on "Wireless Personal Communications" and also a member of the editorial board of other international journals including IEEE Communications Magazine. He is the Technical Program Committee Chairman of the PIMRC'94 International Symposium held in The Hague, The Netherlands, September 19-23, 1994. He is a Fellow of IEE, a Fellow of the Institution of Electronics \& Telecommunication Engineers, and a Member of the New York Academy of Sciences. He is listed in the U.S. Who's Who in the World. 